\title{
Shear and dielectric spectra in highly viscous liquids
}

\author{
U. Buchenau* \\ Institut für Festkörperforschung, Forschungszentrum Jülich Postfach 1913, D-52425 Jülich, Federal Republic of Germany
}

(Received 26 August 2010; revised manuscript received 14 January 2011; published 28 February 2011)

\begin{abstract}
The recently proposed asymmetry model for the highly viscous flow suggests the equality of the dielectric spectrum with the retardation part of the shear spectrum. The equality was checked using literature data, taken under carefully controlled conditions to ensure the same samples and the same temperature control in both measurements. The relation is valid in two substances at all measured temperatures. In two other substances, one can argue that there are good reasons for the deviations that one finds.
\end{abstract}

DOI: 10.1103/PhysRevB.83.052201

PACS number(s): 64.70.P-, 77.22.Gm

A promising approach for the study of the flow process in highly viscous liquids is its comparison ${ }^{1-6}$ in different techniques. One usually finds the dielectric absorption peak close to the heat-capacity one,${ }^{1-4}$ but the shear modulus peak is about half a decade higher in frequency. In a broad distribution of relaxation times, a modulus peak always appears at a higher frequency than a susceptibility (compliance) peak. ${ }^{7,8}$ The question is whether this is the reason here.

It is easy to convince oneself that the inversion of the dielectric susceptibility to a dielectric modulus does not solve the problem. If the difference between the static and the high-frequency dielectric susceptibility is small compared to the latter, as in the cases discussed in the present Brief Report, one gets practically no peak shift. If it is large, as in the cases of glycerol and propylene carbonate, ${ }^{2}$ one gets a peak shift by more than two decades, much too large to explain the observed difference.

The inversion of the shear modulus $G(\omega)$ is not trivial, because its low-frequency limit is zero. In fact, one has two equivalent textbook descriptions of the shear spectrum of a liquid, ${ }^{7}$ a relaxation spectrum $H(\tau)$ for the description of the complex shear modulus $G(\omega)$ ( $\tau$ relaxation time),

$$
G(\omega)=\int_{-\infty}^{\infty} \frac{H(\tau) i \omega \tau}{1+i \omega \tau} d \ln \tau,
$$

and a retardation spectrum $L(\tau)$ for the description of the complex shear compliance,

$$
J(\omega)=\frac{1}{G}+\int_{-\infty}^{\infty} \frac{L(\tau)}{1+i \omega \tau} d \ln \tau-\frac{i}{\omega \eta} .
$$

Here $G$ is the infinite frequency shear modulus and $\eta$ is the viscosity. A third material constant hidden in this equation is the recoverable compliance $J_{e}^{0}$, the elastic compliance plus the integral over the retardation processes,

$$
J_{e}^{0}=\frac{1}{G}+\int_{-\infty}^{\infty} L(\tau) d \ln \tau .
$$

Equation (2) makes a separation of two independent contributions to the compliance: the retardation spectrum and the viscosity. From our gradually growing understanding of the highly viscous liquid, $, 9,10$ we know that both parts must come from thermally activated transitions between inherent states, stable structures corresponding to minima of the potentialenergy landscape. The retardation spectrum is due to back jumps into the initial inherent state, the viscosity is due to no-return processes. The retardation description separates these two influences, the relaxation description does not.

It has been argued that the Zwanzig-Mori formalism requires a relaxation description, ${ }^{11}$ with the bath processes contributing both to relaxation and to viscosity. A different picture results from the asymmetry model, ${ }^{12}$ based on experimental evidence for a strong asymmetry of the secondary relaxation. ${ }^{13}$ In this picture, the viscous flow occurs when a distorted inner core domain does not jump back into its undistorted ground state, but relaxes by the relaxation processes of the surroundings. This implies separate viscosity and relaxation processes, so the retardation scheme should apply. Also, the viscous flow should not be accompanied by a dielectric signal, because the molecules of the inner domain do not change their orientation in the flow process. Thus one expects the equality of the dielectric spectrum with the shear retardation spectrum in the asymmetry model.

It is interesting to consider a liquid with no back-jump preference. For a stationary flow in such a liquid, the removal of the constant shear stress would only result in the immediate recoverable compliance $1 / G$, because there is no reason for a further back flow. There would be no retardation spectrum; the Maxwell time $\tau_{M}=\eta / G$ would be the only relaxation time and $G J_{e}^{0}=1$.

In a real liquid, one finds an extremely broad spectrum of relaxation times and measured values ${ }^{14}$ of $G J_{e}^{0}$ of the order of 3. Numerical simulations ${ }^{10}$ also find a large back-jump probability for the thermally activated transitions between the inherent states. Thus it is always possible to define a normalized shear retardation susceptibility $\chi_{s r}$,

$$
\chi_{s r}(\omega)=\frac{G}{G J_{e}^{0}-1} \int_{-\infty}^{\infty} \frac{L(\tau)}{1+i \omega \tau} d \ln \tau .
$$

$\chi_{s r}$ is normalized with respect to the compliance step; the function begins with the value 1 at low frequency and ends with 0 at high frequency.

With $J(\omega)=1 / G(\omega)$ and Eq. (2), one can translate Eq. (4) to

$$
\chi_{s r}(\omega)=\frac{1}{G J_{e}^{0}-1}\left(\frac{G}{G(\omega)}-1+\frac{i}{\omega \tau_{M}}\right) .
$$

On the dielectric side, one can pragmatically ${ }^{15}$ define a normalized dielectric susceptibility

$$
\chi_{\epsilon}(\omega)=\frac{\epsilon(\omega)-\epsilon_{\text {high }}}{\Delta \epsilon},
$$


where $\epsilon_{\text {high }}$ is the real part of the dielectric constant in the $\mathrm{GHz}$ range and $\Delta \epsilon=\epsilon_{s}-\epsilon_{\text {high }}\left(\epsilon_{s}\right.$ is the static susceptibility of the liquid). This is again normalized, beginning with the value 1 at low frequency and going down to zero at high frequency. The comparison is done for four samples with a relatively small $\Delta \epsilon$ (smaller than 0.15): TPE (triphenylethylene), squalane, 1,4-polybutadiene, and DC704, a silicon oil used in diffusion pumps. 5

One way to compare is to fit $G(\omega)$, then calculate the shear retardation susceptibility from this fit and compare to the dielectric measurement. The fit is better than the data themselves, because small deviations in $G(\omega)$ at low $\omega$ tend to explode in the calculated retardation spectrum (see, for example, the increasing error bars in Figs. 3 and 4 of Schröter and Donth's paper ${ }^{2}$ ). The fit also supplies the three parameters $G, \eta$, and $J_{e}^{0}$ needed for the calculation of the normalized shear retardation susceptibility. If one restricts oneself to a comparison of the imaginary parts, $\Delta \epsilon$ is the only parameter which has to be adapted to the dielectric data.

The procedure does not remove the accuracy problem at low frequency completely; the position of the resulting shear susceptibility $\alpha$ peak remains rather sensitive to the fit parameters for $G(\omega)$. The physical reason for this is the viscosity, which overshadows the low-frequency relaxations in a shear measurement (but not in a dielectric measurement). Here, $G(\omega)$ was modeled in terms of the asymmetry model, ${ }^{12}$ introducing as an extra parameter a width $w$ of the cutoff at the critical barrier $V_{c}$ in order to get the best possible fit.

In TPE, the first example, the relation is fulfilled within experimental error. This is seen in Fig. 1. Figure 1(a) shows the fit to $G(\omega)$, Fig. 1(b) the comparison of the dielectric to

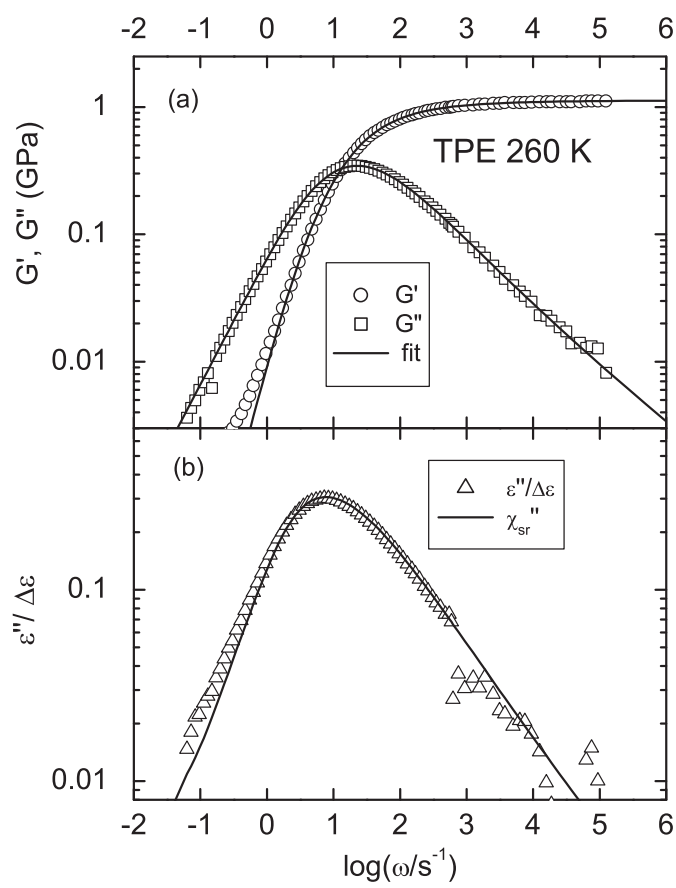

FIG. 1. Validity of the relation in triphenylethylene (TPE). (a) Fit of dynamical shear data (Ref. 5) at $260 \mathrm{~K}$. (b) Comparison of the measured dielectric damping to the shear retardation damping at the same temperature.

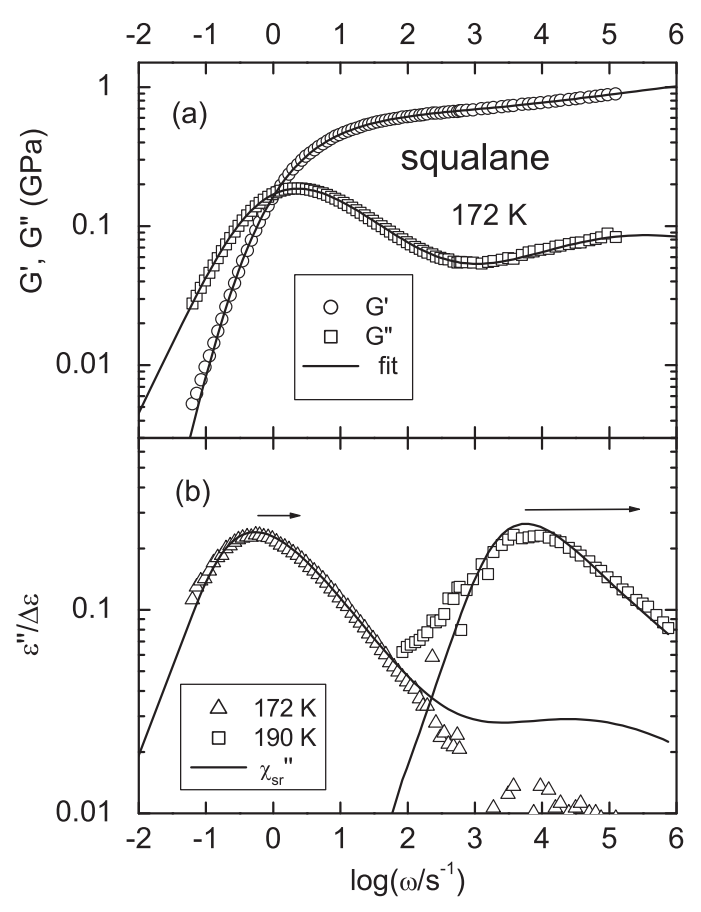

FIG. 2. Validity of the relation in squalane. (a) Fit of dynamical shear data (Ref. 5) at $172 \mathrm{~K}$. (b) Comparison of the measured dielectric damping at 172 and $190 \mathrm{~K}$ to the shear retardation damping at the two temperatures, respectively. The arrows show the positions of the peak in $G^{\prime \prime}(\omega)$.

the shear retardation susceptibility. Other temperatures yield the same result.

The same good agreement is found in squalane, our second example. Figure 2(a) shows again the fit to $G(\omega)$ at $172 \mathrm{~K}$, Fig. 2(b) shows the comparison between dielectric and shear retardation susceptibility damping at two temperatures, 172 and $190 \mathrm{~K}$. This case is particularly impressive, because the shift between shear modulus and dielectric peak increases from half a decade at the glass transition to one and a half at higher temperatures. Nevertheless, one finds good agreement at all temperatures, though the secondary peak is clearly weaker in dielectrics than in shear.

The third case, DC704 in Fig. 3, does not show the perfect agreement of the two previous cases. The shear retardation susceptibility $\alpha$ peak lies a bit to the right of the dielectric one. However, one also finds a slightly higher negative slope in the $\epsilon^{\prime \prime}$ data, indicating a decrease of the ratio of the coupling constants of the elementary relaxations to dielectrics and shear with increasing frequency. If one corrects with a decrease of $6 \%$ per frequency decade, one recovers a good fit. Again, the result does not depend on temperature.

The last case, 1,4-polybutadiene (more precisely, a mixture of $80 \%$ 1,4-polybutadiene and 20\% 1,2-polybutadiene) in Fig. 4 shows strong differences, with the shear retardation susceptibility peak an order of magnitude slower than the dielectric one. Here, the deviations are much too pronounced to be repaired by the simple scaling which worked for DC704. However, this complete breakdown is in fact understandable. 1,4-polybutadiene consists of two kinds of monomers, cis and trans, with the cis-monomers responsible for the dielectric signal. A recent thorough simulation study ${ }^{20}$ of 1,4-polybutadiene 


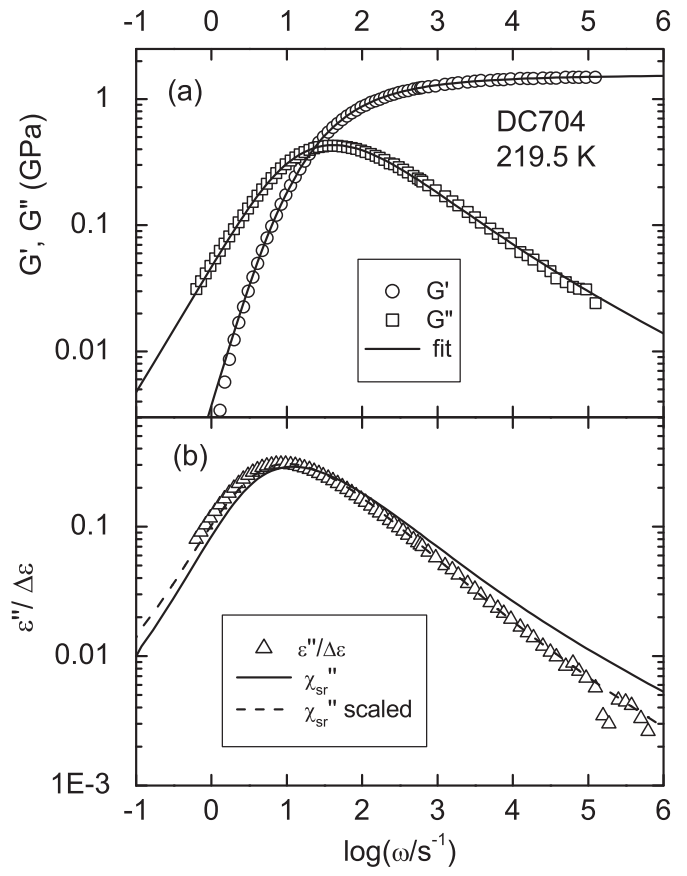

FIG. 3. Peak shift in DC704 (a) Fit of dynamical shear data (Ref. 5) at 219.5 K. (b) Comparison of the measured dielectric damping to the shear retardation damping at the same temperature (continuous line). The dashed line is obtained assuming a decrease of the dielectrics-shear coupling ratio by $6 \%$ per frequency decade.

finds that their response is decidedly faster than the one of the trans monomers.

Our findings are not completely independent on our choice of a model ${ }^{12}$ for $G(\omega)$; taking other models, one

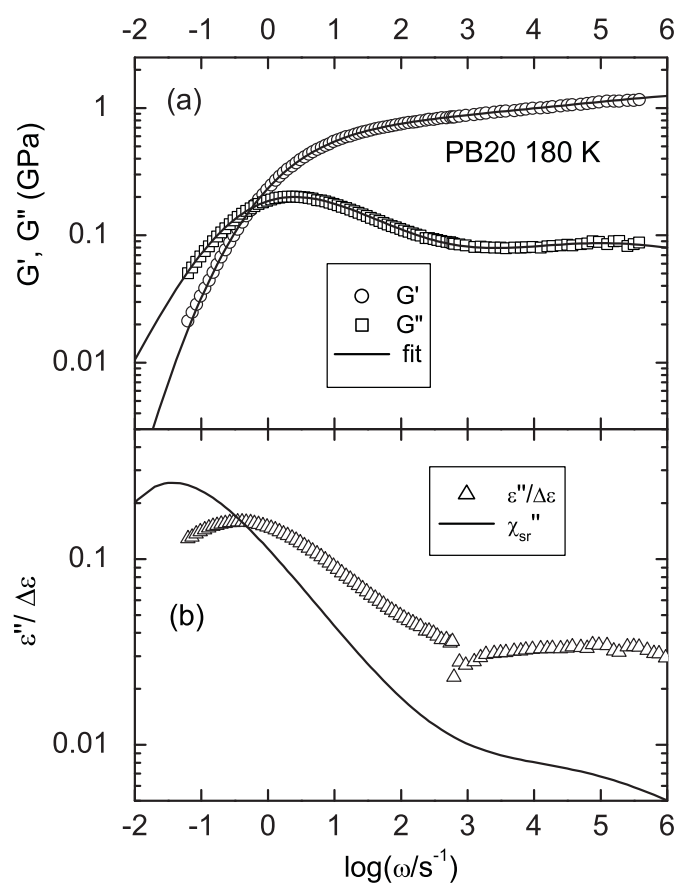

FIG. 4. Strong differences in polybutadiene. (a) Fit of dynamical shear data (Ref. 5) at $180 \mathrm{~K}$. (b) Comparison of the measured dielectric damping to the shear retardation damping at the same temperature. can even fit $G(\omega)$ with a diverging recoverable compliance. ${ }^{5}$ However, the results can be checked independently by an alternative procedure. One can fit the dielectric data first with a Havriliak-Negami function (adding a Cole-Cole function for the secondary peak if necessary). With this fit, one calculates a normalized dielectric susceptibility from Eq. (6). Then, one can check whether this susceptibility describes the $G(\omega)$ data correctly according to Eq. (5), taking not only $G, \eta$, and $J_{e}^{0}$ as free parameters, but also the time constant of the HavriliakNegami function. If this agrees with the one in the dielectric fit within experimental error (about a tenth of a decade) and if the fit is satisfactory, one again concludes that the shear retardation and dielectric fluctuation spectra agree. In our four cases, this procedure corroborated the results from the $G(\omega)$ fit, thus supporting the choice of a shear model with a finite recoverable compliance.

Note that the identity of dielectric and shear retardation spectra found in two of our four cases has a different reason from the one of energy and density fluctuation spectra in strongly correlated liquids ${ }^{21,22}$ with a Prigogine-Defay ratio close to 1 . In that case, the identity of the spectra is due to the strong correlation of the fluctuations. In our case, dielectric and shear fluctuations are not correlated at all, because a vector cannot be correlated with a tensor for symmetry reasons. Equal spectra do not necessarily imply strong correlation, though strong correlation implies equal spectra.

The equality of the spectra must rather result from the decay mechanism. If the local strain disappears completely in a transition between two inherent states, the same must be true for the local dipole moment. In particular, the full decay of the shear strain should be accompanied by a full decay of the dielectric polarization (counterexamples are the normal modes in polymers ${ }^{23}$ or the monoalcohols ${ }^{24}$ ).

Summarizing the experimental and numerical evidence, one has two substances with identical shear retardation and dielectric spectra at all measured temperatures within experimental accuracy and two other substances where one has good reasons to attribute the deviations to the frequency dependence of the coupling ratio. It seems to be the generic situation in glass-forming liquids that the spectra agree. Nevertheless, for particular materials microscopic peculiarities may cause significant deviations like the one in 1,4-polybutadiene.

The finding provides a solid basis for the detailed comparison of the two most important techniques in the study of highly viscous liquids. If the spectra do not agree, one can search for the microscopic reason. If the spectra agree, the two techniques are complementary. The dielectric data provide the exact shape of the retardation spectrum, in particular at the low-frequency end, which is not well seen in the shear measurement. The shear data supply the three material constants, shear modulus, viscosity, and recoverable compliance, which together with the spectrum describe the flow. Such a description opens up the exciting possibility to look for relations between the three material constants and the spectrum, to see whether one can find a new concept of the flow process, hopefully even one in which the viscosity emerges quantitatively out of the spectrum. 
The paper profited a lot from intense discussions with the Roskilde group, in particular Niels Boie Olsen, Bo Jakobsen, Kristine Niss, Tage Christensen, Albena Nielsen, and Tina Hecksher. Also, very helpful discussions with Robert Pick, Reiner Zorn, and Andreas Wischnewski are gratefully acknowledged. *buchenau-juelich@t-online.de

${ }^{1}$ K. L. Ngai and R. W. Rendell, Phys. Rev. B 41, 754 (1990).

${ }^{2}$ K. Schröter and E. Donth, J. Non-Cryst. Solids 307-310, 270 (2002).

${ }^{3}$ S. Corezzi, M. Beiner, K. Huth, K. Schröter, S. Capaccioli, R. Casalini, D. Fioretto, and E. Donth, J. Chem. Phys. 117, 2435 (2002).

${ }^{4}$ L. Carpentier, O. Bastin, and M. Descamps, J. Phys. D 35, 402 (2002).

${ }^{5}$ K. Niss, B. Jakobsen, and N. B. Olsen, J. Chem. Phys. 123, 234510 (2005); B. Jakobsen, K. Niss, and N. B. Olsen, ibid. 123, 234511 (2005); data available at the website [http://glass.ruc.dk/data/].

${ }^{6}$ C. Gainaru, O. Lips, A. Troshagina, R. Kahlau, A. Brodin, F. Fujara, and E. A. Rössler, J. Chem. Phys. 128, 147505 (2008).

${ }^{7}$ D. J. Ferry, Viscoelastic Properties of Polymers, 3rd ed. (John Wiley, New York, 1980), Chap. 3.

${ }^{8}$ E. Donth, Relaxation and Thermodynamics in Polymers (Akademie-Verlag, Berlin, 1992), p. $31 \mathrm{ff}$.

${ }^{9}$ P. G. Debenedetti and F. H. Stillinger, Nature (London) 410, 259 (2001).

${ }^{10}$ A. Heuer, J. Phys.: Condens. Matter 20, 373101 (2008).

${ }^{11} \mathrm{R}$. Pick (private communication). The argument is based on the fact that a mechanical modulus can be calculated directly from the Zwanzig-Mori formalism, while a mechanical compliance cannot. For details, see T. Franosch, A. Latz and R. Pick, Eur. Phys. J. B 31, 229 (2003); T. Franosch and R. Pick, ibid. 47, 341 (2005).

${ }^{12}$ U. Buchenau, J. Chem. Phys. 131, 074501 (2009).
${ }^{13}$ J. C. Dyre and N. B. Olsen, Phys. Rev. Lett. 91, 155703 (2003).

${ }^{14}$ D. J. Plazek, C. H. Bero, and I.-C. Chay, J. Non-Cryst. Solids 172-174, 181 (1994).

${ }^{15}$ One should be aware of possible problems with this definition. The first is the difference between external and internal fields (Ref. 16), which requires more complicated expressions like the one of Fatuzzo and Mason (Ref. 17). For this reason, the present work restricts itself to examples with a small $\Delta \epsilon$, where the Fatuzzo-Mason expression equals the simple Eq. 8 given here within experimental error. The second possible problem is the exclusion of high-frequency contributions of the molecular rotation (Refs. 5 , 18, and 19), which are in principle part of the dielectric spectrum and will contribute as well to the shear spectrum. The definition of Eq. (6) excludes this high-frequency part of the spectra.

${ }^{16} \mathrm{G}$. Williams, Chem. Rev. 72, 55 (1972).

${ }^{17}$ E. Fatuzzo and P. R. Mason, Proc. Phys. Soc. 90, 729 (1967).

${ }^{18}$ T. Keyes and D. Kievelson, J. Chem. Phys. 54, 1786 (1971).

${ }^{19}$ C. Dreyfus, A. Aouadi, R. M. Pick, T. Berger, A. Patkowski, and W. Steffen, Eur. J. Phys. B 9, 401 (1999).

${ }^{20}$ A. Narros, A. Arbe, F. Alvarez, J. Colmenero, and D. Richter, J. Chem. Phys. 128, 224905 (2008).

${ }^{21}$ N. P. Bailey, U. R. Pedersen, N. Gnan, T. B. Schroeder, and J. C. Dyre, J. Chem. Phys. 129, 184507 (2008).

${ }^{22}$ T. B. Schroeder, N. P. Bailey, U. R. Pedersen, N. Gnan, and J. C. Dyre, J. Chem. Phys. 131, 234503 (2009).

${ }^{23}$ K. Adachi and T. Kotaka, Macromolecules 18, 466 (1985).

${ }^{24}$ B. Jakobsen, C. Maggi, T. Christensen, and J. C. Dyre, J. Chem. Phys. 129, 184502 (2008). 